\title{
Lidocaine toxicity in a student undergoing upper gastrointestinal endoscopy
}

Background - A young medical student developed severe toxicity, including seizures, respiratory distress, hypotension, and asystole, and died after gargling with lidocaine before upper gastrointestinal endoscopy. Upper gastrointestinal endoscopy is usually a safe outpatient procedure before which the throat is often anaesthetised.

Case report-A 21 year old medical student presented with the symptoms of acid peptic disease and was referred for outpatient oesophagogastroduodenoscopy. He was of average build, did not smoke, and did not take any drugs; he had no history of respiratory or cardiac disease. The patient was advised to fast overnight before the procedure, and on the day of the procedure his pulse, blood pressure, and respiratory rate were normal. He was asked to gargle with $20 \mathrm{ml} \mathrm{4 \%}$ lidocaine solution ( $800 \mathrm{mg}$ of the drug) for 60 seconds, and was advised not to swallow the drug. While gargling he developed seizures and respiratory distress. We performed endotracheal intubation and he was given oxygen, intravenous midozolam, and hydrocortisone $500 \mathrm{mg}$. His condition did not improve and he subsequently developed hypotension and severe bradycardia that changed rapidly to asystole; despite all further measures, he died.

Discussion-Excessive dosage, rapid absorption, or accidental intravascular injection of local anaesthetics may affect the patient adversely, but an allergic reaction is rare. High doses of such anaesthetics can also lead to central nervous system and cardiovascular toxicity. Damage to the central nervous system may cause numbness of the tongue and perioral area, restlessness, and ultimately seizures, respiratory failure, and coma. Cardiovascular toxicity may develop subsequently and profound bradycardia and asystole sometimes also occur. ${ }^{1}$

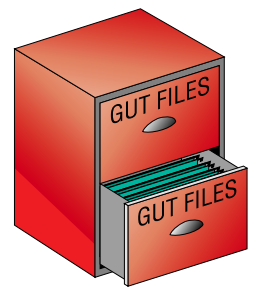

Our patient received $800 \mathrm{mg}$ lidocaine, much more than the recommended maximum dose $(200 \mathrm{mg})$. Gargling with lidocaine is common in many countries-for example, Pakistan, where metered dose sprays are not available. A literature search using Medline did not find any reports of deaths from lidocaine toxicity before oesophagogastroduodenoscopy, although there have been cases of toxicity from the local use of lidocaine before endotracheal intubation. ${ }^{23}$ Conclusion-Lidocaine is safe for local anaesthesia if the dose is $<200 \mathrm{mg}^{4}$ and, although we advise that lidocaine should not be swallowed when gargling, it seems clear that the drug still enters the gastrointestinal tract making it difficult to control the amount of drug administered. Thus, centres using the gargling technique are strongly advised to change to metered dose sprays that deliver an exact quantity of lidocaine; $10 \mathrm{mg}$ of lidocaine is given per spray and thus, the total dose administered to the patient can be controlled more easily.

B F ZUBERI
M R SHAIKH
N-U-N JATOI
W M SHAIKH
Chandka Medical College, Larkana
Pakistan

Correspondence to: Dr Bader Faiyaz Zuberi, 5 Professors' Colony, Chandka Medical College Larkana, Pakistan. email: bader@workmail.com

1 Naguib M, Magboul MM, Samarkandi AH, et al. Adverse effects and drug interactions associated with local and regional anaesthesia. Drug Saf interactions associ.

2 Resar LM, Helfaer MA. Recurrent seizures in a neonate after lidocaine administration. F Perinatol 1998;18:193-5.

3 Smith M, Wolfram W, Rose R. Toxicity seizures in an infant caused by (or related to) oral viscous lidocaine use. $\mathcal{F}$ Emerg Med 1992;10:587-90.

4 Walker IA, Slovis CM. Lidocaine in the treatment of status epilepticus. Acad Emerg Med 1997;4:918-22. 